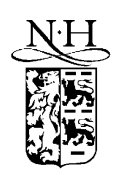

\title{
Implications of a new effective chiral meson Lagrangian
}

\author{
Alexander A. Osipov ${ }^{1}$, Marcos Sampaio, Brigitte Hiller* \\ Centro de Física Teórica, Departamento de Física da Universidade de Coimbra, 3004-516 Coimbra, Portugal
}

Received 13 July 2001; revised 23 October 2001; accepted 14 November 2001

\begin{abstract}
Based on a recently derived effective chiral meson Lagrangian from the extended $S U(2) \otimes S U(2)$ Nambu-Jona-Lasinio (ENJL) model, in the linear realization of chiral symmetry, we extract to leading order in the $1 / N_{c}$ expansion all associated relevant three-point functions $\rho \rightarrow \pi \pi, \sigma \rightarrow \pi \pi$, $a_{1} \rightarrow \rho \pi, a_{1} \rightarrow \sigma \pi$, as well as the amplitude for $\pi \pi$ scattering. We discuss the formal differences of these amplitudes as compared with those derived in the literature and calculate the associated decay widths and scattering parameters. The differences have two origins:

(i) new terms, which are proportional to the current quark mass and arise from taking the correct NJL vacuum from the first steps in a proper-time expansion, are present in the Lagrangian;

(ii) an implemented chiral covariant treatment of the diagonalization in the pseudoscalar-axialvector sector induces new couplings between three or more mesonic fields.

Both effects have been derived from the chiral Ward Takahashi identities, which are fully taken into account at each order of the proper-time expansion. ( 2001 Elsevier Science B.V. All rights reserved.
\end{abstract}

PACS: $12.39 . \mathrm{Fe} ; 11.30 . \mathrm{Rd}$

Keywords: Chiral meson Lagrangian; Correct vacuum and proper-time expansion

\section{Introduction}

The Nambu-Jona-Lasinio (NJL) model [1] and its several extensions (see, e.g., [2-14]) have been vastly studied as effective models of the strong interaction, based on the chiral dynamics of four-quark interactions. By incorporating the main symmetries of QCD and being reminiscent of the effective four-fermion interaction for QCD, obtained after eliminating the gluonic degrees of freedom [15], the NJL model is a useful playground for simulating relevant features of low-energy hadron physics. Its innumerous applications

\footnotetext{
* Corresponding author.

E-mail address: brigitte@ teor.fis.uc.pt (B. Hiller).

${ }^{1}$ On leave from the Laboratory of Nuclear Problems, JINR, 141980 Dubna, Russia.
} 
range from the calculation of the low-lying meson spectra, meson couplings, decay and scattering amplitudes, diquark physics and extensions to the baryonic sector, to modeling of finite density and temperature effects on chiral properties of hadrons.

In the present paper, we focus on the implications of a recently derived $S U(2) \otimes$ $S U$ (2) effective chiral Lagrangian [18,19], on the low-lying hadron phenomenology. The Lagrangian has been constructed on the basis of the ENJL model by using the Schwinger proper-time representation for the modulus of the one-loop quark determinant [16,17], and the following long wavelength expansion of its heat kernel. This semiclassical WKB expansion of the ENJL action is implemented by polynomial counterterms, which result from requiring that the symmetry-breaking pattern of the fermionic Lagrangian in the presence of the explicit chiral symmetry-breaking term be equivalent to the one of the bosonized effective Lagrangian [19,20]. As a consequence of these symmetry requirements we have shown how relevant and previously not considered current quark-mass terms appear in the local action of the chiral mesonic fields. These terms allow to account for the correct vacuum already at the first steps of the proper-time expansion and lead to a resummation in the current quark mass. Furthermore, in the case of the linear realization of chiral symmetry, the Lagrangian contains new meson couplings which derive from a modified diagonalization of the axial-vector-pseudoscalar interaction, which we have shown to be necessary in order to preserve the chiral transformation properties of the vector mesons. We consider worthwhile understanding the consequences of such new structures on relevant amplitudes and scattering processes. As we shall show, the effects of the new current quark-mass terms will be manifest in all considered amplitudes, and may appear both explicitly and implicitly through the coupling parameters. As for the covariant diagonalization, we shall observe the following:

(1) the $\rho \pi \pi$ coupling becomes a three-derivative type, contrary to the one-derivative type obtained in the usual linear approaches. This is important, since the latter violates chiral symmetry [21]. On the mass shell one recovers the one-derivative structure.

(2) The $a_{1} \pi \sigma$ coupling acquires also new three-derivative type of couplings. On the mass shell it reduces in form to the known results of [12,22].

(3) The amplitudes, $\sigma \pi \pi$ and $a_{1} \rho \pi$ are not altered by the proposed covariant diagonalization.

(4) The contact term with four pion fields gets modified with extra two- and fourderivatives in the fields.

One expects therefore that when off-shell processes are at work, such as in form factors or $\rho$ exchange in $\pi \pi$ scattering, the related amplitudes are affected correspondingly. We shall show, however, that in the case of $\pi \pi$ scattering, one recovers old results (up to current quark-mass terms), regardless of using the covariant diagonalization.

We work in the leading $1 / N_{c}$ approximation, that is, to fermion one-loop level. The bosonized Lagrangian is correspondingly treated to tree-level order in the meson couplings. Furthermore, we sum the proper-time series up to the third Seeley-DeWitt coefficient, which amounts to keeping, out of the full momentum dependence of the $n$-point functions, only the quadratic and logarithmic divergent contributions. There are several reasons to 
stop at this order in the heat kernel expansion. First, once this is done, the masses and coupling constants of the chiral fields are completely fixed in a way which guarantees that the first and the second Weinberg sum rules are automatically satisfied. Second, truncating the proper-time series at this order is substantiated by the results of [23], where an infinite number of local counterterm operators were added to the ENJL Lagrangian, with couplings fixed, such that the corresponding Adler function exhibited the properties of the "lowest meson dominance" approximation to large- $N_{c}$ QCD. For the vector and axial-vector twopoint functions this requirement was tantamount to removing the nonconfining terms and guarantees their correct matching to the QCD short-distance behaviour. Third, one might expect, except for amplitudes which are finite previous to regularization, the divergent contributions to dominate over the finite ones.

The paper is structured as follows. In the second section, we set up the notation and situate the problem, by giving a short review of the results obtained in $[18,19]$. In Section 3 , we derive the amplitudes $\rho \rightarrow \pi \pi, \sigma \rightarrow \pi \pi, a_{1} \rightarrow \rho \pi$ and $a_{1} \rightarrow \sigma \pi$ and discuss the differences with respect to similar amplitudes obtained from other models based on ENJLtype Lagrangians. In Section 4, we derive the amplitude for $\pi \pi$ scattering. In Section 5 , we present the numerical results. We conclude with a summary and outlook.

\section{The Lagrangian: current quark-mass terms and covariant diagonalization}

The starting point is the effective quark Lagrangian of strong interactions which is invariant under a global colour $S U\left(N_{c}\right)$ symmetry:

$$
\begin{aligned}
\mathcal{L}= & \bar{q}\left(\mathrm{i} \gamma^{\mu} \partial_{\mu}-m_{c}\right) q+\frac{G_{S}}{2}\left[(\bar{q} q)^{2}+\left(\bar{q} \mathrm{i} \gamma_{5} \tau_{i} q\right)^{2}\right] \\
& -\frac{G_{V}}{2}\left[\left(\bar{q} \gamma^{\mu} \tau_{i} q\right)^{2}+\left(\bar{q} \gamma^{\mu} \gamma_{5} \tau_{i} q\right)^{2}\right] .
\end{aligned}
$$

Here $q$ is a flavor doublet of Dirac spinors for quark fields $\bar{q}=(\bar{u}, \bar{d})$. Summation over the colour indices is implicit. We use the standard notation for the isospin Pauli matrices $\tau_{i}$. The current quark-mass matrix $m_{c}=\operatorname{diag}\left(\widehat{m}_{u}, \widehat{m}_{d}\right)$ is chosen in such a way that $\widehat{m}_{u}=$ $\widehat{m}_{d}=\widehat{m}$. Without this term the Lagrangian (1) would be invariant under global chiral $S U(2) \otimes S U(2)$ symmetry. The coupling constants $G_{S}$ and $G_{V}$ have dimensions (Length) ${ }^{2}$ and can be fixed from the meson-mass spectrum. The transformation law for the quark fields is the following:

$$
\delta q=\mathrm{i}\left(\alpha+\gamma_{5} \beta\right) q, \quad \delta \bar{q}=-\mathrm{i} \bar{q}\left(\alpha-\gamma_{5} \beta\right),
$$

where parameters of global infinitesimal chiral transformations are chosen as $\alpha=\alpha_{i} \tau_{i}$, $\beta=\beta_{i} \tau_{i}$. Under infinitesimal chiral transformations the Lagrangian $\mathcal{L}$ exhibits, therefore, the following explicit symmetry-breaking pattern:

$$
\delta \mathcal{L}=-2 \mathrm{i} \widehat{m}\left(\bar{q} \gamma_{5} \beta q\right),
$$

which is to be kept intact at each stage of calculations (here we are not considering the anomalous sector). The chiral effective Lagrangian which we obtain [19] from (1) as 
result of the heat kernel expansion up to and including the third-order Seeley-DeWitt coefficient and taking into account the symmetry requirements has the following form in the spontaneously broken phase:

$$
\begin{aligned}
\mathcal{L}_{\mathrm{eff}}= & \frac{v_{\mu i}^{2}+a_{\mu i}^{2}}{2 G_{V}}-\frac{\widehat{m}\left(\sigma^{2}+\vec{\pi}^{2}\right)}{2(m-\widehat{m}) G_{S}}-\frac{N_{c} J_{1}}{8 \pi^{2}}\left[\frac{1}{6} \operatorname{tr}\left(v_{\mu \nu}^{2}+a_{\mu \nu}^{2}\right)\right. \\
& \left.-\frac{1}{2} \operatorname{tr}\left(\left(\nabla_{\mu} \pi\right)^{2}+\left(\nabla_{\mu} \sigma\right)^{2}\right)+\left(\sigma^{2}+2(m-\widehat{m}) \sigma+\pi_{i}^{2}\right)^{2}\right],
\end{aligned}
$$

where the trace is to be taken in isospin space. Here we have used the notation:

$$
\begin{aligned}
& v_{\mu \nu}=\partial_{\mu} v_{\nu}-\partial_{\nu} v_{\mu}-\mathrm{i}\left[v_{\mu}, v_{\nu}\right]-\mathrm{i}\left[a_{\mu}, a_{\nu}\right], \\
& a_{\mu \nu}=\partial_{\mu} a_{\nu}-\partial_{\nu} a_{\mu}-\mathrm{i}\left[a_{\mu}, v_{\nu}\right]-\mathrm{i}\left[v_{\mu}, a_{\nu}\right], \\
& \nabla_{\mu} \sigma=\partial_{\mu} \sigma-\mathrm{i}\left[v_{\mu}, \sigma\right]+\left\{a_{\mu}, \pi\right\}, \\
& \nabla_{\mu} \pi=\partial_{\mu} \pi-\mathrm{i}\left[v_{\mu}, \pi\right]-\left\{a_{\mu}, \sigma+m-\widehat{m}\right\},
\end{aligned}
$$

with $v_{\mu}=v_{\mu i} \tau_{i}, a_{\mu}=a_{\mu i} \tau_{i}, \sigma, \pi=\pi_{i} \tau_{i}$ designating the vector-isovector, axial-vectorisovector, scalar-isoscalar and pseudoscalar-isovector fields, respectively, and $m$ is the constituent quark mass. In terms of these fields, the infinitesimal chiral transformation laws read:

$$
\begin{array}{ll}
\delta \sigma=-\{\beta, \pi\}, & \delta \pi=\mathrm{i}[\alpha, \pi]+2(\sigma+m-\widehat{m}) \beta, \\
\delta v_{\mu}=\mathrm{i}\left[\alpha, v_{\mu}\right]+\mathrm{i}\left[\beta, a_{\mu}\right], & \delta a_{\mu}=\mathrm{i}\left[\alpha, a_{\mu}\right]+\mathrm{i}\left[\beta, v_{\mu}\right] .
\end{array}
$$

The variation of the second term of (4) yields the symmetry-breaking pattern of the Lagrangian in terms of the collective fields, which is the equivalent of Eq. (3) in terms of the fermionic variables:

$$
\delta \mathcal{L}=-\frac{2 \widehat{m}}{G_{S}}\left(\beta_{i} \pi_{i}\right)=\delta \mathcal{L}_{\text {eff }}
$$

All other terms in (4) are chiral invariant. The function $J_{1}$ appearing in (4) is one of the set of integrals $J_{n}$ emerging in the heat kernel expansion [19],

$$
J_{n}\left(m^{2}, \Lambda^{2}\right)=\int_{0}^{\infty} \frac{\mathrm{d} T}{T^{2-n}} \mathrm{e}^{-T m^{2}} \rho\left(T, \Lambda^{2}\right), \quad n=0,1,2, \ldots
$$

In the explicit evaluation of these integrals, we use as regulating kernel the Pauli-Villars cutoff [24] with two subtractions

$$
\rho\left(T, \Lambda^{2}\right)=1-\left(1+T \Lambda^{2}\right) \mathrm{e}^{-T \Lambda^{2}} .
$$

Prior to regularization, the $J_{1}$ integral is logarithmically divergent. The other characteristic divergence of the ENJL model at one-loop order is the quadratic one, given by $J_{0}$, which has been traded by the gap equation in writing down (4)

$$
\frac{m-\widehat{m}}{m G_{S}}=\frac{N_{c} J_{0}}{2 \pi^{2}}
$$

to establish the real vacuum of the spontaneously broken phase. As it stands, the effective Lagrangian (4) still requires a diagonalization of the pseudoscalar-axial-vector fields 
appearing in the quadratic forms for the covariant derivatives. We have shown in [19] that the simplest replacement of variables which fulfills the linear transformation property (10) not only for old variables, $v_{\mu}, a_{\mu}$, but also for new ones, $v_{\mu}^{\prime}, a_{\mu}^{\prime}$, is

$$
\begin{aligned}
& a_{\mu}=a_{\mu}^{\prime}+\frac{\kappa}{2}\left(\left\{\sigma+m-\widehat{m}, \partial_{\mu} \pi\right\}-\left\{\pi, \partial_{\mu} \sigma\right\}\right), \\
& v_{\mu}=v_{\mu}^{\prime}+\frac{\mathrm{i} \kappa}{2}\left(\left[\sigma, \partial_{\mu} \sigma\right]+\left[\pi, \partial_{\mu} \pi\right]\right) .
\end{aligned}
$$

For the case at hand, the commutator $\left[\sigma, \partial_{\mu} \sigma\right]=0$. These redefinitions involve new terms that are bilinear in the fields and induce changes at the level of couplings with three or more fields, as compared to the noncovariant diagonalizations that have widely been used previously in the linear chiral symmetry versions of the ENJL model. The replacement (15) is identical to the field redefinition considered in [12,35] for the case of nonlinear realization of chiral symmetry. The constant $\kappa$ is fixed by the requirement that the bilinear part of the effective Lagrangian becomes diagonal in the fields $\pi$ and $a_{\mu}^{\prime}$. We find in this way that

$$
\frac{1}{2 \kappa}=(m-\widehat{m})^{2}+\frac{\pi^{2}}{N_{c} J_{1} G_{V}} .
$$

The physical meson fields are obtained as usual by bringing the kinetic terms to their standard form. For the vector fields, one has:

$$
v_{\mu}^{\prime}=\sqrt{\frac{6 \pi^{2}}{N_{c} J_{1}}} v_{\mu}^{(p h)} \equiv \frac{g_{\rho}}{2} v_{\mu}^{(p h)}, \quad a_{\mu}^{\prime}=\frac{g_{\rho}}{2} a_{\mu}^{(p h)} .
$$

Then we have

$$
m_{\rho}^{2}=\frac{6 \pi^{2}}{N_{c} J_{1} G_{V}}, \quad m_{a}^{2}=m_{\rho}^{2}+6(m-\widehat{m})^{2} .
$$

In particular, it implies the relations

$$
g_{A}=1-\frac{6(m-\widehat{m})^{2}}{m_{a}^{2}}=\frac{m_{\rho}^{2}}{m_{a}^{2}}, \quad \kappa=\frac{3}{m_{a}^{2}} .
$$

We also have to redefine the spin-0 fields:

$$
\sigma=\sqrt{\frac{4 \pi^{2}}{N_{c} J_{1}}} \sigma^{(p h)} \equiv g_{\sigma} \sigma^{(p h)}, \quad \pi=g_{\pi} \pi^{(p h)}, \quad g_{\pi}=\frac{g_{\sigma}}{\sqrt{g_{A}}} .
$$

The mass formulae for spin-0 fields are

$$
m_{\pi}^{2}=\frac{\widehat{m} g_{\pi}^{2}}{(m-\widehat{m}) G_{S}}, \quad m_{\sigma}^{2}=g_{A} m_{\pi}^{2}+4(m-\widehat{m})^{2} .
$$

As compared with previous calculations in [5,12], our mass formulae have a different dependence on the current quark mass.

Let us also point out that after the field redefinitions the symmetry-breaking part takes the form [25]

$$
\delta \mathcal{L}_{\mathrm{eff}}=-2 m_{\pi}^{2} f_{\pi} \beta_{i} \pi_{i}^{(p h)},
$$


which leads to the well known PCAC relation for the divergence of the quark axial-vector current

$$
\partial_{\mu} \vec{J}_{A}^{\mu}=2 f_{\pi} m_{\pi}^{2} \vec{\pi}^{(p h)} .
$$

We used the relation

$$
g_{\pi}=\frac{m-\widehat{m}}{f_{\pi}}
$$

to get (22).

\section{Three-meson vertices}

\subsection{The $\sigma \pi \pi$ interaction}

After using the field redefinitions (15) in the effective Lagrangian (4) and collecting all terms involving one scalar and two pseudoscalar fields, one gets

$$
\mathcal{L}_{\sigma \pi \pi}=-2 \frac{g_{\sigma}}{g_{A}}(m-\widehat{m}) \sigma\left\{\left[1-\frac{m_{\pi}^{2}\left(1-g_{A}\right)}{2(m-\widehat{m})^{2}}\right] \vec{\pi}^{2}+\frac{1-g_{A}^{2}}{2(m-\widehat{m})^{2}}\left(\partial_{\mu} \vec{\pi}\right)^{2}\right\},
$$

where use has been made of the mass relation for the scalar field Eq. (21), the field renormalizations (20) (here and henceforth we drop the index ( $p h)$ on the physical fields), and the mixing parameter $\kappa$, Eq. (16). One obtains for the decay $\sigma(q) \rightarrow \pi_{a}(p) \pi_{b}\left(p^{\prime}\right)$ ( $a, b$ are isospin indices) the amplitude

$$
\begin{aligned}
& \mathcal{M}_{\sigma \pi \pi}\left(p, p^{\prime}\right)=\frac{1}{4} \operatorname{Tr}\left\{\tau_{a}, \tau_{b}\right\} f_{\sigma \pi \pi}\left(p, p^{\prime}\right) . \\
& f_{\sigma \pi \pi}\left(p, p^{\prime}\right)=4 \frac{g_{\sigma}}{g_{A}}(m-\widehat{m})\left\{1-\frac{1-g_{A}}{2(m-\widehat{m})^{2}}\left[m_{\pi}^{2}+p p^{\prime}\left(1+g_{A}\right)\right]\right\} .
\end{aligned}
$$

On the mass shell, $m_{\sigma}^{2}=2\left(m_{\pi}^{2}+p p^{\prime}\right)$ and using (21) for the $\sigma$ mass, one obtains

$$
f_{\sigma \pi \pi}=4 g_{\sigma}(m-\widehat{m})\left\{g_{A}+\frac{m_{\pi}^{2}\left(1-g_{A}\right)^{2}}{4(m-\widehat{m})^{2}}\right\} \text {. }
$$

This amplitude differs from previously calculated ones by the current quark-mass terms. For instance in [13]: keeping only the logarithmically divergent integrals at zero squared momentum, we find a correspondence to the considered order of the present heat kernel expansion, after the substitutions ((lhs) are the notations of [13] and (rhs) the present)

$$
m \rightarrow m-\widehat{m}, \quad \delta=1-\frac{6 m^{2}}{m_{a}^{2}} \rightarrow g_{A},
$$

which lead to Eq. (28). The decay width is obtained in the standard way:

$$
\Gamma_{\sigma \pi \pi}=\frac{3 f_{\sigma \pi \pi}^{2}}{8 \pi m_{\sigma}^{2}} \sqrt{\left(m_{\sigma}^{2}-4 m_{\pi}^{2}\right)}
$$




\subsection{The $\rho \pi \pi$ interaction}

Again by collecting all terms involving the $v_{\mu}$-meson field and two-pseudoscalar fields, after the redefinition (15), one obtains the interaction Lagrangian:

$$
\begin{aligned}
\mathcal{L}_{\rho \pi \pi}= & \mathrm{i} g_{\rho} g_{\pi}^{2} \operatorname{tr}\left\{\frac{\kappa}{8 G_{V}} v_{\mu}\left[\pi, \partial_{\mu} \pi\right]-\frac{N_{c} J_{1}}{16 \pi^{2}}\left[\frac{\kappa}{3}\left(1-\kappa(m-\widehat{m})^{2}\right) \tilde{v}_{\mu \nu}\left[\partial_{\mu} \pi, \partial_{\nu} \pi\right]\right.\right. \\
& \left.\left.-\left(2 \kappa(m-\widehat{m})^{2}-1\right) \partial_{\mu} \pi\left[v_{\mu}, \pi\right]\right]\right\},
\end{aligned}
$$

with $\tilde{v}_{\mu \nu}$ denoting the derivative terms of (5). The terms containing $\kappa$ which are not multiplying quark-mass factors stem from the proposed field bilinears in the redefinition (15) for the vector fields and were therefore absent in previous analyzes. Also all $\widehat{m}$ terms are new. Using Eqs. (16) and (19), one can recast the interaction in the form

$$
\mathcal{L}_{\rho \pi \pi}=-\mathrm{i} \frac{g_{\rho}\left(1+g_{A}\right)}{8 m_{\rho}^{2}} \operatorname{tr}\left(\tilde{v}_{\mu \nu}\left[\partial_{\mu} \pi, \partial_{\nu} \pi\right]\right) .
$$

The interaction is of three-derivative type, as opposed to the usual one-derivative coupling. This is a consequence of the chiral covariant diagonalization. On the mass shell, one obtains, after partial integration in the action and discarding total derivatives:

$$
\mathcal{L}_{\rho \pi \pi}=-\mathrm{i} \frac{g_{\rho}}{8}\left(1+g_{A}\right) \operatorname{tr}\left(v_{\mu}\left[\pi, \partial_{\mu} \pi\right]\right) .
$$

The Lagrangian becomes on-shell equivalent in form to the standard expression, for the nonlinear as well as linear cases, see, e.g., $[12,13]$. In order to make these comparisons, one should again keep only the logarithmically divergent contributions in the cases considered in $[12,13]$, to be compatible with the order of the heat kernel expansion considered in the present approach. Starting from the Lagrangian $\mathcal{L}_{\rho \pi \pi}$ of [12],

$$
\begin{aligned}
& \mathcal{L}_{\rho \pi \pi}=\frac{-\mathrm{i} g_{V}}{2 \sqrt{2}} \operatorname{tr}\left(V_{\mu \nu}\left[\xi^{\mu}, \xi^{\nu}\right]\right), \\
& \xi_{\mu}=\mathrm{i}\left(\xi^{+} \partial_{\mu} \xi-\xi \partial_{\mu} \xi^{+}\right) \rightarrow \frac{1}{f_{\pi}} \partial_{\mu} \pi+\cdots, \quad \xi=\exp \left(\frac{-\mathrm{i}}{2 f_{\pi}} \lambda_{i} \phi_{i}\right),
\end{aligned}
$$

one has the following correspondence between the notation of [12] (lhs) and the present one (rhs):

$$
\begin{aligned}
& g_{V}=\frac{N_{c}}{48 \pi^{2} f_{V}}\left(1-g_{A}^{2}\right) \Gamma(0, x) \rightarrow \frac{1-g_{A}^{2}}{2 g_{\rho}}, \quad f_{V}^{2}=\frac{N_{c} \Gamma(0, x)}{24 \pi^{2}} \rightarrow \frac{1}{g_{\rho}^{2}}, \\
& \Gamma(0, x) \rightarrow J_{1}, \quad g_{A} \rightarrow \frac{m_{\rho}^{2}}{m_{a_{1}}^{2}},
\end{aligned}
$$

where $x=m^{2} / \Lambda_{\chi}^{2}$. In the expression for $g_{V}$ of [12] we have already dropped a term proportional to $\Gamma(1, x)$, which would correspond to a $J_{2}$ integral in our notation and therefore be of higher order than the one considered in the heat kernel expansion of the present work. Note that, although there is a formal equivalence to the standard 
result, hidden information stemming from the current quark-mass terms is carried by the expressions relating $m_{\rho}$ to $m_{a_{1}}$ and by $g_{A}$, Eqs. (18) and (19).

The amplitude for the process $\rho_{\mu}^{a}(q) \rightarrow \pi^{b}(p) \pi^{c}\left(p^{\prime}\right)$ is

$$
\mathcal{M}_{\rho \pi \pi}\left(p, p^{\prime}\right)=\frac{1}{4} \operatorname{Tr}\left(\tau_{a}\left[\tau_{b}, \tau_{c}\right]\right)\left(p-p^{\prime}\right)_{\mu} \epsilon^{\mu}(q) f_{\rho \pi \pi},
$$

where $\epsilon^{\mu}(q)$ is the polarization of the vector particle and

$$
f_{\rho \pi \pi}=\frac{g_{\rho}}{2}\left(1+g_{A}\right)
$$

for on-shell particles. The decay width is then

$$
\Gamma_{\rho \pi \pi}=\frac{\left|\vec{p}_{c}\right|^{3}}{6 \pi m_{\rho}^{2}} f_{\rho \pi \pi}^{2},
$$

with $\vec{p}_{c}$ being the center of mass momentum of the pions, $\left|\vec{p}_{c}\right|=\sqrt{\left(m_{\rho}^{2}-4 m_{\pi}^{2}\right)} / 2$.

\subsection{The $a_{1} \rho \pi$ and $a_{1} \sigma \pi$ interactions}

These processes are interesting in relation to the branching ratio $\operatorname{Br}\left(a_{1} \rightarrow \pi(\pi \pi)_{s}\right)$. According to Weinberg [26], chiral symmetry arguments lead to the prediction $\operatorname{Br}\left(a_{1} \rightarrow\right.$ $\left.\pi(\pi \pi)_{s}\right)=10-15 \%$, in conflict with the value quoted in Particle Data until 1996 [27]. The main source of the $(\pi \pi)_{s}$ pairs is the scalar particle decay and the main decay channel for $a_{1}$ is $a_{1} \rightarrow \rho \pi$. The ratio of these two decay modes for the $a_{1}$ should then represent a reasonable estimate of the branching ratio.

The Lagrangians for the couplings $a_{1} \rho \pi$ and $a_{1} \sigma \pi$ are obtained in a similar way as in the previous cases:

$$
\begin{aligned}
& \mathcal{L}_{a_{1} \rho \pi}=\mathrm{i} f_{\pi} \frac{g_{\rho}^{2}}{4 g_{A}} \operatorname{tr}\left\{\frac{\kappa}{3}\left(a_{\mu}\left[\partial_{\nu} \pi, \tilde{v}_{\mu \nu}\right]+v_{\mu}\left[\partial_{\nu} \pi, \tilde{a}_{\mu \nu}\right]\right)+a_{\mu}\left[v_{\mu}, \pi\right]\right\}, \\
& \mathcal{L}_{a_{1} \sigma \pi}=g_{\rho} \frac{1-g_{A}}{\sqrt{g_{A}}} \operatorname{tr}\left\{\sigma a_{\mu} \partial_{\mu} \pi+\frac{1}{12(m-\widehat{m})^{2}} \tilde{a}_{\mu \nu}\left(\partial_{\mu} \pi \partial_{\nu} \sigma-\partial_{\nu} \pi \partial_{\mu} \sigma\right)\right\},
\end{aligned}
$$

with $\tilde{a}_{\mu \nu}$ the derivative terms contained in (6). Contrary to the case of $\mathcal{L}_{\rho \pi \pi}$ interaction, the terms bilinear in the fields in Eq. (15) do not contribute to $\mathcal{L}_{a_{1} \rho \pi}$. The terms proportional to $\kappa$ stem only from the linear combination $\kappa(m-\widehat{m}) \partial_{\mu} \pi$ of the shift in the $a_{\mu}$ field. On the mass shell, the interaction Lagrangians reduce to

$$
\mathcal{L}_{a_{1} \rho \pi}=\mathrm{i} f_{\pi} \frac{g_{\rho}^{2}}{4} \operatorname{tr}\left(a_{\mu}\left[v_{\mu}, \pi\right]\right),
$$

which coincides in form with the results in $[12,22,28]$ and to

$$
\mathcal{L}_{a_{1} \sigma \pi}=-g_{\rho} \sqrt{g_{A}} \operatorname{tr}\left(\sigma a_{\mu} \partial_{\mu} \pi\right),
$$

which corresponds to the result of $[12,22]$. Let us note, however, that the couplings $g_{A}$ and $f_{\pi}$ depend, in the present approach, on extra current quark-mass terms, Eqs. (19) and (24). 
The decay amplitudes for the processes $a_{1 \mu}^{a}(q) \rightarrow \rho_{\nu}^{b}(p) \pi^{c}\left(p^{\prime}\right)$ and $a_{1 \mu}^{a}(q) \rightarrow$ $\sigma(p) \pi^{b}\left(p^{\prime}\right)$ on-shell are

$$
\begin{aligned}
& \mathcal{M}_{a_{1} \rho \pi}\left(p, p^{\prime}\right)=-\frac{\mathrm{i}}{4} \operatorname{Tr}\left(\tau_{a}\left[\tau_{b}, \tau_{c}\right]\right) \epsilon_{\mu}(q) \epsilon_{\nu}^{*}(p) f_{a_{1} \rho \pi}^{\mu \nu}, \\
& \mathcal{M}_{a_{1} \sigma \pi}\left(p, p^{\prime}\right)=\frac{\mathrm{i}}{4} \operatorname{Tr}\left\{\tau_{a}, \tau_{b}\right\} \epsilon_{\mu}(q) p^{\prime \mu} f_{a_{1} \sigma \pi},
\end{aligned}
$$

with

$$
f_{a_{1} \rho \pi}^{\mu \nu}=f_{\pi} g_{\rho}^{2} g^{\mu \nu} \quad \text { and } \quad f_{a_{1} \sigma \pi}=2 g_{\rho} \sqrt{g_{A}} .
$$

Finally, the decay widths are calculated to be

$$
\Gamma_{a_{1} \rho \pi}=\frac{f_{\pi}^{2} g_{\rho}^{4}}{12 \pi m_{a}^{3}}\left(2+\frac{(q p)^{2}}{m_{a}^{2} m_{\rho}^{2}}\right) \sqrt{(q p)^{2}-m_{a}^{2} m_{\rho}^{2}}
$$

with $2 q p=m_{a}^{2}+m_{\rho}^{2}-m_{\pi}^{2}$ and

$$
\Gamma_{a_{1} \sigma \pi}=\frac{m_{a}}{192 \pi}\left|f_{a_{1} \sigma \pi}\right|^{2}\left\{\left[1-\left(\frac{m_{\sigma}+m_{\pi}}{m_{a}}\right)^{2}\right]\left[1-\left(\frac{m_{\sigma}-m_{\pi}}{m_{a}}\right)^{2}\right]\right\}^{3 / 2} .
$$

\section{4. $\pi \pi$ scattering}

The scattering amplitude $T_{a b ; c d}$ for the process $\pi^{a}\left(p_{1}\right)+\pi^{b}\left(p_{2}\right) \rightarrow \pi^{c}\left(p_{3}\right)+\pi^{d}\left(p_{4}\right)$ has the well-known isotopic structure:

$$
T_{a b ; c d}(s, t, u)=\delta_{a b} \delta_{c d} A(s, t, u)+\delta_{a c} \delta_{b d} A(t, s, u)+\delta_{a d} \delta_{c b} A(u, t, s) .
$$

Here, the standard Mandelstam variables for two-particle elastic scattering, $s, t$ and $u$, are defined by

$$
s=\left(p_{1}+p_{2}\right)^{2}, \quad t=\left(p_{1}-p_{2}\right)^{2}, \quad u=\left(p_{1}-p_{4}\right)^{2} .
$$

Amplitudes with definite isospin $(I), T^{I}$, are then

$$
\begin{aligned}
& T^{0}(s, t, u)=A(t, s, u)+A(u, t, s)+A(s, t, u), \\
& T^{1}(s, t, u)=A(t, s, u)-A(u, t, s), \\
& T^{2}(s, t, u)=A(t, s, u)+A(u, t, s) .
\end{aligned}
$$

After the redefinitions (15), the Lagrangian (4) contributes with scalar and $\rho$-meson exchange as well as with a contact term to the scattering amplitude. First, we evaluate the scalar-exchange amplitude $A_{\sigma}(s, t, u)$ using the interaction Lagrangian (25) for the $\sigma \pi \pi$ vertex:

$$
\begin{aligned}
A_{\sigma}(s, t, u)=\frac{16 g_{\pi}^{2}(m-\widehat{m})^{2}}{g_{A}\left(m_{\sigma}^{2}-s\right)}\{ & {\left[1-\frac{m_{\pi}^{2}\left(1-g_{A}\right)}{2(m-\widehat{m})^{2}}\right]^{2} } \\
& -\left[1-\frac{m_{\pi}^{2}\left(1-g_{A}\right)}{2(m-\widehat{m})^{2}}\right] \frac{\left(1-g_{A}^{2}\right)\left(s-2 m_{\pi}^{2}\right)}{(m-\widehat{m})^{2}} \\
& \left.+\frac{\left(1-g_{A}^{2}\right)^{2}\left(s-2 m_{\pi}^{2}\right)^{2}}{16(m-\widehat{m})^{4}}\right\} .
\end{aligned}
$$


The scalar propagator is expanded up to a desired order in $(m-\widehat{m})^{-2}$,

$$
\begin{aligned}
\frac{1}{m_{\sigma}^{2}-s} & =\frac{1}{4(m-\widehat{m})^{2}}\left[1+\frac{g_{A} m_{\pi}^{2}-s}{4(m-\widehat{m})^{2}}\right]^{-1} \\
& =\frac{1}{4(m-\widehat{m})^{2}}\left\{1-\frac{g_{A} m_{\pi}^{2}-s}{4(m-\widehat{m})^{2}}+\frac{\left(g_{A} m_{\pi}^{2}-s\right)^{2}}{16(m-\widehat{m})^{4}}+\cdots\right\},
\end{aligned}
$$

leading to

$$
A_{\sigma}(s, t, u)=4 \frac{g_{\pi}^{2}}{g_{A}}+\frac{g_{A}}{f_{\pi}^{2}}\left[s\left(2-\frac{1}{g_{A}^{2}}\right)-m_{\pi}^{2}\left(4-\frac{3}{g_{A}}\right)\right]+\cdots .
$$

Next, we obtain the $\rho$-exchange amplitude $A_{\rho}(s, t, u)$ using the interaction Lagrangian (31) for the $\rho \pi \pi$ vertex. The $\rho$-propagator has the conventional form

$$
\Delta_{\mu \nu}^{a b}\left(x_{1}-x_{2}\right)=-\mathrm{i} \delta_{a b} \int \frac{\mathrm{d}^{4} k}{(2 \pi)^{4}}\left(\frac{k_{\mu} k_{\nu}}{m_{\rho}^{2}}-g_{\mu \nu}\right) \frac{\mathrm{e}^{-\mathrm{i} k\left(x_{1}-x_{2}\right)}}{\left(m_{\rho}^{2}-k^{2}-\mathrm{i} \epsilon\right)}
$$

and the amplitude reads

$$
A_{\rho}(s, t, u)=\frac{g_{\rho}^{2}}{m_{\rho}^{4}}\left(1+g_{A}\right)^{2}\left\{\frac{t^{2}(s-u)}{m_{\rho}^{2}-t}+\frac{u^{2}(s-t)}{m_{\rho}^{2}-u}\right\} .
$$

This amplitude starts at $\mathcal{O}\left(p^{6}\right)$ in chiral counting and therefore does not contribute to the Weinberg result. It can be compared to the $\rho$-meson-exchange contribution to the $\pi \pi$ scattering amplitude derived by Gasser and Leutwyler [21], in spite of the fact that their $\rho$ meson has origin in an antisymmetric tensor field. This is because in the evaluation of the respective $S$-matrix element, $\mathcal{M}_{4 \pi}$, with $\rho$ exchange

$$
\begin{aligned}
\mathcal{M}_{4 \pi}= & \frac{-\mathrm{i} g_{\rho}^{2}\left(1+g_{A}^{2}\right)}{128 m_{\rho}^{2}}\left(\delta_{i l} \delta_{j m}-\delta_{i m} \delta_{j l}\right) \int \mathrm{d}^{4} x_{1} \mathrm{~d}^{4} x_{2}\left\langle\pi^{c}\left(p_{3}\right) \pi^{d}\left(p_{4}\right)\right| \partial_{\mu} \pi_{i}\left(x_{1}\right) \\
& \times \partial_{\nu} \pi_{j}\left(x_{1}\right) \partial_{\alpha} \pi_{l}\left(x_{2}\right) \partial_{\beta} \pi_{m}\left(x_{2}\right) \mathcal{U}_{\mu \nu \alpha \beta}\left|\pi^{a}\left(p_{1}\right) \pi^{b}\left(p_{2}\right)\right\rangle,
\end{aligned}
$$

one encounters the term $\mathcal{U}_{\mu \nu \alpha \beta}$ :

$$
\mathcal{U}_{\mu \nu \alpha \beta}=\int \frac{\mathrm{d}^{4} k}{(2 \pi)^{4}} \frac{\mathrm{e}^{-\mathrm{i} k\left(x_{1}-x_{2}\right)}}{\left(m_{\rho}^{2}-k^{2}\right)}\left[-k_{\mu} k_{\alpha} g_{\nu \beta}+k_{\nu} k_{\alpha} g_{\mu \beta}-k_{\nu} k_{\beta} g_{\mu \alpha}+k_{\mu} k_{\beta} g_{\nu \alpha}\right],
$$

which is identical to a piece of the Green's function for the $\rho$ meson in [21] and leads to the contribution proportional to $\left(m_{\rho}^{2}-k^{2}\right)^{-1}$ for the corresponding $\pi \pi$-scattering amplitude.

Finally, we evaluate the contact term, by collecting all terms with four pseudoscalar fields:

$$
\begin{aligned}
\mathcal{L}_{c}=g_{\pi}^{4} \operatorname{tr}\{ & \frac{-\kappa^{2}}{16 G_{V}}\left[\pi, \partial_{\mu} \pi\right]^{2}+\frac{N_{c} J_{1}}{8 \pi^{2}}\left[\frac{\kappa^{2}}{6}\left(1-\kappa(m-\widehat{m})^{2}\right)^{2}\left[\partial_{\mu} \pi, \partial_{\nu} \pi\right]^{2}-\frac{\pi^{4}}{2}\right. \\
& +\frac{\kappa^{2}}{2}(m-\widehat{m})^{2}\left\{\partial_{\mu} \pi, \pi\right\}^{2}+\frac{\kappa}{2} \partial_{\mu} \pi\left[\left[\pi, \partial_{\mu} \pi\right], \pi\right] \\
& \left.\left.+\kappa^{2}(m-\widehat{m})^{2} \partial_{\mu} \pi\left[\pi,\left[\pi, \partial_{\mu} \pi\right]\right]\right]\right\} .
\end{aligned}
$$


All terms containing powers of $\kappa$ as factors (except the ones multiplying quark masses) derive from the covariant diagonalization and were not present in previous schemes. After some rearrangement, one can write the contact term as

$$
\begin{aligned}
\mathcal{L}_{c}= & -\frac{g_{\pi}^{2}}{2 g_{A}}\left(\vec{\pi}^{2}\right)^{2}+\frac{\left(1-g_{A}\right)}{2 g_{A} f_{\pi}^{2}}\left[\left(\vec{\pi} \partial_{\mu} \vec{\pi}\right)^{2}-g_{A} \vec{\pi}^{2}\left(\partial_{\mu} \vec{\pi}\right)^{2}\right] \\
& -\left(1-g_{A}^{2}\right)\left(1+g_{A}\right) \frac{\left(\partial_{\mu} \vec{\pi} \times \partial_{\nu} \vec{\pi}\right)^{2}}{4 f_{\pi}^{2} m_{\rho}^{2}}
\end{aligned}
$$

which leads to the contact amplitude

$$
\begin{aligned}
A_{c}(s, t, u)= & -4 \frac{g_{\pi}^{2}}{g_{A}}+\frac{\left(1-g_{A}\right)}{f_{\pi}^{2} g_{A}}\left[s+2 g_{A}\left(s-2 m_{\pi}^{2}\right)\right] \\
& +\frac{\left(1-g_{A}^{2}\right)\left(1+g_{A}\right)}{4 f_{\pi}^{2} m_{\rho}^{2}}[(s-t) u+(s-u) t] .
\end{aligned}
$$

Now we obtain the complete amplitude $A(s, t, u)$ for $\pi \pi$ scattering, by assembling the scalar- and vector-exchange amplitudes and contact terms. We give here explicitly the result to $p^{4}$ th order:

$$
\begin{aligned}
A(s, t, u)= & \frac{1}{f_{\pi}^{2}}\left(s-m_{\pi}^{2}\right)+\frac{1}{24 g_{A} f_{\pi}^{2}(m-\widehat{m})^{2}}\left\{\left(1-g_{A}^{2}\right)^{2}[(s-t) u+(s-u) t]\right. \\
& \left.+6 g_{A}^{2}\left[\left(s-2 m_{\pi}^{2}\right) g_{A}+m_{\pi}^{2}\right]^{2}\right\}+\mathcal{O}\left(p^{6}\right) .
\end{aligned}
$$

This result can be compared with the one obtained using the noncovariant diagonalization in [13] after keeping there only the logarithmic divergent contributions at zero squared momentum, again to relate to the order of heat kernel expansion considered in the present work. To order $p^{2}$, we obtain the Weinberg result [29]. In fact we find that for any $p^{2}$ order of $A(s, t, u)$ one recovers the previous result, except for the current quark-mass terms (i.e., if one puts everywhere in (61) $m-\widehat{m} \rightarrow m$ ). It turns out that in the case of the usual noncovariant diagonalization and induced linear derivative $\rho$ coupling to the pions, a judicious combination of the chiral noncovariant terms emerging in the vector channel and the contact term simulates the correct structure of the contact term obtained in the case of the covariant diagonalization, up to $p^{4}$ order (the vector-exchange only starts at $p^{6}$ order). Starting from $\mathcal{O}\left(p^{6}\right)$, the vector-exchange term coincides in the two approaches and the contact term does not contribute in both cases, at the considered order of the heat kernel expansion.

In the next section, we analyze the numerical effects due to the present heat kernel expansion on $\pi \pi$ threshold parameters, as compared to the studies where the full momentum expansion of Feynman amplitudes was considered [13]. We do not expect large deviations, since, at least to the order of the heat kernel expansion considered here, the $\pi \pi$ amplitude does not get modified by the covariant diagonalization, and the current quark mass may not be large enough to make the new terms in the amplitude numerically significant. However, it is worthwhile measuring the numerical effects related to the momentum expansions in the two approaches, since they differ by finite nonvanishing 
contributions present in [13], due to differences of logarithmic divergent integrals of different arguments and other finite terms.

\section{Numerical results}

We start the numerical section by calculating the decay widths of the heavy mesons. The four parameters of the model, $G_{S}, G_{V}, \Lambda$ and $\widehat{m}$, are obtained by fixing $m_{\pi}=139 \mathrm{MeV}$, $f_{\pi}=92 \mathrm{MeV}, m_{\rho}=770 \mathrm{MeV}$ and the ratio $g_{A}=m_{\rho}^{2} / m_{a}^{2}$, which we take at two different $m_{a}$ values. For $g_{A}=0.5$, in accordance with the choice of [26], we obtain $m_{a}=1089 \mathrm{MeV}$, $m=314 \mathrm{MeV}, \widehat{m}=1.7 \mathrm{MeV}, G_{S}=3.22 \mathrm{GeV}^{-2}, G_{V}=14.77 \mathrm{GeV}^{-2}, \Lambda=1.536 \mathrm{GeV}$, and for $g_{A}=0.374$, which corresponds to the empirical $m_{a}=1260 \mathrm{MeV}$, we get $m=$ $408 \mathrm{MeV}, \widehat{m}=1.4 \mathrm{MeV}, G_{S}=3.40 \mathrm{GeV}^{-2}, G_{V}=18.49 \mathrm{GeV}^{-2}, \Lambda=1.544 \mathrm{GeV}$. In Table 1 we display the mesonic observables, set I corresponding to the smaller $m$ value and set II to the large one. Some comments are in order here. The decay width $\Gamma_{\sigma \pi \pi}$ and $\Gamma_{\rho \pi \pi}$ turn out to be smaller than the empirical values by roughly a factor two, if one insists on keeping the correct empirical fit for $m_{\pi}$ and $f_{\pi}$. This trend did not change as compared to the calculations in a full momentum scheme, for the cases in which the $\rho$ meson is a well-defined bound state below the quark-antiquark pair threshold; the $\sigma$ meson in the latter case is always slightly embedded in the continuum (with a very small decay width in quark-antiquark pairs [14]). The results for the branching ratio

$$
\operatorname{Br}\left[a_{1} \rightarrow \pi(\pi \pi)_{s}\right] \sim \frac{\Gamma_{a_{1} \rightarrow \sigma \pi}}{\Gamma_{a_{1} \rightarrow \sigma \pi}+\Gamma_{a_{1} \rightarrow \rho \pi}} \sim 14 \% \text { (I); 5\%(II) }
$$

are in fair agreement to the $10-20 \%$ obtained by Weinberg. The rather large change observed in the width $\Gamma_{a_{1} \rightarrow \rho \pi}$ from set II of parameters to I is mainly dictated by the square root term in Eq. (46), which is reduced by roughly a factor two and the change in the coupling $g_{\rho}^{2}$, which gets smaller by $\sim 25 \%$.

Table 1

Some meson properties calculated in the present version of the NJL model are compared to experimental data [34]

\begin{tabular}{cccc}
\hline$[\mathrm{MeV}]$ & Model (set I) & Model (set II) & Experiment \\
\hline$f_{\pi}$ & $92^{*}$ & $92^{*}$ & 93.3 \\
$m_{\pi}$ & $139^{*}$ & $139^{*}$ & 139 \\
$m_{\sigma}$ & 633 & 818 & $400-1200$ \\
$m_{\rho}$ & $770^{*}$ & $770^{*}$ & 770 \\
$m_{a_{1}}$ & $1089^{*}$ & $1260^{*}$ & 1260 \\
$\Gamma_{\sigma \rightarrow \pi \pi}$ & 409 & 394 & $600-1000$ \\
$\Gamma_{\rho \rightarrow \pi \pi}$ & 82 & 86 & 150 \\
$\Gamma_{a 1 \rightarrow \rho \pi}$ & 192 & 420 & seen; full $\Gamma=250-600$ \\
$\Gamma_{a 1 \rightarrow \sigma \pi}$ & 31 & 23 & seen \\
\hline
\end{tabular}

* The asterisks indicate quantities which served as input to determine the model parameters. 
Table 2

The calculated $\pi \pi$-scattering lengths and effective ranges are compared to Soft Meson Theorems (SMT) [29] and experimental data (taken from [30,31], see text please)

\begin{tabular}{cccccccc}
\hline$a_{l}^{\mathrm{I}}$ & $\mathcal{O}\left(p^{4}\right)[\mathrm{I}]$ & $\mathcal{O}\left(p^{6}\right)[\mathrm{I}]$ & $\mathcal{O}\left(p^{4}\right)[\mathrm{II}]$ & $\mathcal{O}\left(p^{6}\right)[\mathrm{II}]$ & Full [13] & SMT & Experiment [30] \\
\hline$a_{0}^{0}$ & 0.166 & 0.167 & 0.161 & 0.162 & 0.17 & 0.16 & $0.26 \pm 0.05$ \\
$b_{0}^{0}$ & 0.188 & 0.192 & 0.178 & 0.179 & 0.19 & 0.18 & $0.25 \pm 0.03$ \\
$a_{0}^{2}$ & -0.0454 & -0.0454 & -0.0454 & -0.0454 & -0.047 & -0.0454 & $-0.028 \pm 0.012$ \\
$b_{0}^{2}$ & -0.0875 & -0.0875 & -0.0875 & -0.0875 & -0.090 & -0.089 & $-0.082 \pm 0.008$ \\
$a_{1}^{1}$ & 0.0336 & 0.0347 & 0.0338 & 0.0351 & 0.038 & 0.030 & $0.038 \pm 0.002$ \\
$a_{2}^{0} \times 10^{4}$ & 5.92 & 8.198 & 5.034 & 7.476 & 6.9 & & $17 \pm 3$ \\
$a_{2}^{2} \times 10^{4}$ & -0.74 & -1.93 & -1.96 & -3.16 & -2.5 & & $1.3 \pm 3$ \\
\hline
\end{tabular}

Next we present in Table 2 the results for threshold parameters $a_{l}^{\mathrm{I}}, b_{l}^{\mathrm{I}}$ from the representation (61) of the $\pi \pi$-scattering amplitude, as compared to the data of [30]. The more recent analysis of [31] yields $a_{0}^{0}=0.288 \pm 0.012 \pm 0.003$ and $a_{0}^{2}=-0.036 \pm 0.009$. Let us note that since we are working at meson tree level, the $p^{2}$ expansion in our case reveals the subjacent quark-antiquark compositeness of the amplitudes and therefore we do not compare it to the meson loop orders related momentum expansion of CHPT (for recent reviews see [32,33]).

At $p^{2}$ order (not shown in the table), the quantities $a_{0}^{0}, b_{0}^{0}, a_{0}^{2}, b_{0}^{2}, a_{1}^{1}$ reproduce the soft pion theorem values. The observed trend of $\pi \pi$-scattering lengths and effective ranges is congruent with the results of the full momentum expansion [13] (included in the table, for the larger value of the constituent quark mass considered there $(m=390 \mathrm{MeV}))$. Some deviations are observed in the $\mathrm{D}$-wave scattering lengths. We have checked that at $p^{8}$ order there are no significant changes for any of the calculated scattering lengths and effective ranges, from which we infer that the differences in the higher partial waves are related to the presence of finite terms in [13], not existent in the heat kernel expansion (see also discussion at the end of previous section).

In the present calculation, the vector exchange has still a noticeable contribution for $a_{1}^{1}$ (the scalar-exchange and contact contributions stabilize at $p^{4}$ order), as well as in $a_{2}^{0}$ and $a_{2}^{2}$.

The case of rather small quark mass $(\simeq 200 \mathrm{MeV})$ also considered in [13] (since it describes better the scalar form factor of the pion) was calculated as well with the present method, leading to similar conclusions as for the large-mass case. Nevertheless, we do not consider further this case here, since the corresponding parameter set yields worse results for the heavy-meson decays.

In the light of the numerical results, the present heat kernel expansion yields comparable results and trends in the momentum expansion for the scattering parameters calculated 
from Feynman amplitudes with a full momentum dependence in the vertices [13], for the $\mathrm{S}$ and $\mathrm{P}$ waves. Some sizeable effects are observed in the higher partial waves.

\section{Summary and outlook}

The main concern of this work was to show the formal and numerical implications of a recently derived effective $S U(2) \otimes S U(2)$ chiral Lagrangian with linear realization of chiral symmetry on mesonic observables: mass spectra, strong decays and $\pi \pi$-scattering parameters. The considered Lagrangian was constructed on the basis of the SchwingerDeWitt proper-time method applied to the ENJL model. The resulting semiclassical WKB expansion of the ENJL action has been done around the correct NJL vacuum state, defined by the corresponding Schwinger-Dyson equation in the case with explicit chiral symmetry breaking. We derive that the amplitudes carry the signature of this vacuum: the amplitudes get relevant current quark-mass corrections, not present in previous approaches. Furthermore, we also derive that amplitudes with three or more fields are affected by the diagonalization in the pseudoscalar-axial-vector sector which was implemented to correctly describe the vector meson chiral transformations for the linear realization of chiral symmetry. We have studied in detail the structure of the amplitudes $\sigma \pi \pi, \rho \pi \pi$, $a_{1} \rho \pi$ and $a_{1} \sigma \pi$ as well as $\pi \pi$ scattering. The $\rho \pi \pi, a_{1} \sigma \pi$ amplitudes and the contact fourpion interaction get modified by the covariant diagonalization and all studied amplitudes depend on current quark-mass terms. On the mass shell, we obtain that all studied processes are not affected by the covariant diagonalization, becoming structurally identical (except for current quark-mass terms) to the ones obtained in the nonlinear as well as linear realizations of chiral symmetry, at the same order of the heat kernel expansion. In this study, the current quark-mass effects are numerically negligible, as expected for the $S U(2)$ case. From the formal point of view, however, the way the new structures appear in the amplitudes hints at possible large numerical deviations for the $S U(3)$ case. The extension to the $S U(3)$ case is presently under study.

\section{Acknowledgements}

This work is supported by grants provided by Fundaçã o para a Ciência e a Tecnologia, PRAXIS/C/FIS/12247/1998, PESO/P/PRO/15127/1999, POCTI/35304/FIS/2000, CERN/ P/FIS/40119/2000, PRAXIS XXI/BDP/22016/99 and NATO "Outreach" Cooperation Program.

\section{References}

[1] V.G. Vaks, A.I. Larkin, Zh. Eksp. Teor. Fiz. 40 (1961) 282;

Y. Nambu, G. Jona-Lasinio, Phys. Rev. 122 (1961) 345;

Y. Nambu, G. Jona-Lasinio, Phys. Rev. 124 (1961) 246.

[2] D. Ebert, M.K. Volkov, Z. Phys. C 16 (1983) 205. 
[3] M.K. Volkov, Ann. Phys. (NY) 157 (1984) 282.

[4] A. Dhar, S. Wadia, Phys. Rev. Lett. 52 (1984) 959;

A. Dhar, R. Shankar, S. Wadia, Phys. Rev. D 31 (1985) 3256.

[5] D. Ebert, H. Reinhardt, Nucl. Phys. B 271 (1986) 188.

[6] M. Wakamatsu, W. Weise, Z. Phys. A 331 (1988) 173.

[7] V. Bernard, R.L. Jaffe, U.-G. Meißner, Nucl. Phys. B 308 (1988) 753.

[8] S. Klimt, M. Lutz, U. Vogl, W. Weise, Nucl. Phys. A 516 (1990) 429.

[9] M. Takizawa, K. Tsushima, Y. Kohyama, K. Kubodera, Nucl. Phys. A 507 (1990) 611.

[10] C. Schüren, E. Ruiz Arriola, K. Goeke, Nucl. Phys. A 547 (1992) 612.

[11] V. Bernard, A.A. Osipov, U.-G. Meißner, Phys. Lett. B 285 (1992) 119.

[12] J. Bijnens, C. Bruno, E. de Rafael, Nucl. Phys. B 390 (1993) 503.

[13] V. Bernard, A.H. Blin, B. Hiller, Y.P. Ivanov, A.A. Osipov, U.-G. Meißner, Ann. Phys. (NY) 249 (1996) 499.

[14] V. Bernard, A.H. Blin, B. Hiller, Y.P. Ivanov, A.A. Osipov, U.-G. Meißner, Phys. Lett. B 409 (1997) 483.

[15] G. 't Hooft, Phys. Rev. D 14 (1976) 3432.

[16] J. Schwinger, Phys. Rev. 82 (1951) 664.

[17] B. DeWitt, Dynamical Theory of Groups and Fields, Gordon and Breach, New York, 1965.

[18] A.A. Osipov, B. Hiller, Phys. Lett. B 488 (2000) 299.

[19] A.A. Osipov, B. Hiller, Phys. Rev. D 62 (2000) 114013.

[20] A.A. Osipov, B. Hiller, Phys. Rev. D 63 (2001) 094009.

[21] J. Gasser, H. Leutwyler, Ann. Phys. (NY) 158 (1984) 142.

[22] V. Bernard, A.A. Osipov, U.-G. Meißner, Phys. Lett. B 292 (1992) 205.

[23] S. Peris, M. Perrottet, E. de Rafael, JHEP 9805 (1998) 011.

[24] W. Pauli, F. Villars, Rev. Mod. Phys. 21 (1949) 434.

[25] S. Gasiorowicz, D.A. Geffen, Rev. Mod. Phys. 41 (1969) 531.

[26] S. Weinberg, Phys. Rev. Lett. 65 (1990) 1177.

[27] Particle Data Group, Phys. Rev. D 50 (1994) 1173.

[28] J. Prades, Z. Phys. C 63 (1994) 491.

[29] S. Weinberg, Phys. Rev. Lett. 18 (1967) 507.

[30] C.D. Froggatt, J.L. Petersen, Nucl. Phys. B 129 (1977) 89.

[31] P. Truol, hep-ex/0012012.

[32] G. Amoròs, J. Bijnens, P. Talavera, hep-ph/0003258.

[33] G. Colangelo, J. Gasser, H. Leutwyler, hep-ph/0103088.

[34] Particle Data Group, Eur. Phys. J. C 15 (2000) 1.

[35] G. Ecker, J. Gasser, H. Leutwyler, A. Pich, E. de Rafael, Phys. Lett. B 223 (1989) 425. 